\title{
Searching for Submillisecond Pulsars from Highly Polarized Radio Sources
}

\author{
J. L. $\operatorname{Han}^{1}$, R. N. Manchester ${ }^{2}$, A. G. Lyne ${ }^{3}$, G. J. Qiao \\ ${ }^{1}$ National Astronomical Observatories, CAS, Beijing 100012, China \\ ${ }^{2}$ Australia Telescope National Facility, CSIRO, Epping, Australia \\ ${ }^{3}$ University of Manchester, Jodrell Bank Observatory, SK11 9DL, UK \\ ${ }^{4}$ Department of Astronomy, Peking University, Beijing 100871, China
}

\begin{abstract}
Pulsars are among the most highly polarized sources in the universe. The NVSS has cataloged 2 million radio sources with linear polarization measurements, from which we have selected 253 sources, with polarization percentage greater than $25 \%$, as targets for pulsar searches. We believe that such a sample is not biased by selection effects against ultra-short spin or orbit periods. Using the Parkes 64-m telescope, we conducted searches with sample intervals of $50 \mu \mathrm{s}$ and $80 \mu \mathrm{s}$, sensitive to submillisecond pulsars. Unfortunately we did not find any new pulsars.
\end{abstract}

\section{Introduction}

The NRAO VLA Sky Survey (NVSS; Condon et al. 1998) contains 1.8 million radio sources with flux density at $1400 \mathrm{MHz}$ greater than $2.5 \mathrm{mJy}$ with linear polarization information. Many pulsars are known to have high linear polarization on average. Han \& Tian (1999) identified 97 known pulsars from the NVSS source catalog and they found that, on average, the linear polarization percentage of pulsars is much higher than that of other classes of objects such as quasars and BL-Lac objects. Therefore, high linear polarization can be used as a criterion for selecting pulsars with no period bias, including submillisecond pulsars for which normal untargeted surveys are difficult. Submillisecond pulsars could be strange-quark stars (e.g. Madsen 1998), but are so far undetected (e.g. Edwards, van Straten \& Bailes 2001).

\section{The Search}

We have selected 253 unresolved sources with high linear polarization (linear polarization percentage $L / S>25 \%$; uncertainty of $L / S<10 \%$ ) from the NVSS catalog and searched for pulsed emission. These sources have a flux density which is generally larger than $\sim 4 \mathrm{mJy}$. To have been missed by previous pulsar surveys, they must have some combination of the following properties: short (millisecond) pulse period, short orbital period, and/or high dispersion. Most of these sources are at relatively high Galactic latitudes and so the first two properties are likely to be the most important if they are pulsars. 


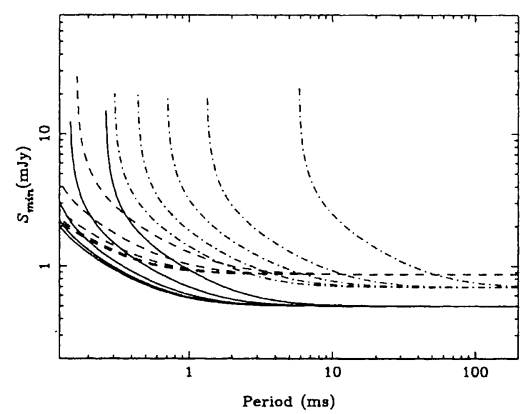

Figure 1. The minimum detectable flux density at $8 \sigma$, assuming the pulse has a width of $10 \%$ of period. Solid lines are for the $512 \times 0.5 \mathrm{MHz}$ system, and dashed lines are for the $256 \times 0.25 \mathrm{MHz}$ system, each with $5 \mathrm{DM}$ values (bottom to top): $0,20,40,80$, and $160 \mathrm{pc} \mathrm{cm}^{-3}$. Dashdotted lines are for the converted sensitivity of the Parkes $70 \mathrm{~cm}$ pulsar survey. Our searches are sensitive to submillisecond pulsars.

We searched for short-period pulsed emission from these selected sources by using the Parkes telescope on 1999 Oct 8-19 and Dec 9-11. The central beam of the $20-\mathrm{cm}$ multibeam system was used, having a system temperature of $21 \mathrm{~K}$ and a gain of $0.735 \mathrm{~K} \mathrm{Jy}^{-1}$. The two orthogonally polarized signals were amplified and fed to a filterbank system, which was used in one of two configurations: 512 channels of $0.5 \mathrm{MHz}$ bandwidth centered at $1390 \mathrm{MHz}$, or 256 channels of $0.25 \mathrm{MHz}$ bandwidth centered on $1362 \mathrm{MHz}$. After detection of each channel, the two polarization signals were added together and 1-bit digitized. Data were sampled every $80 \mu \mathrm{s}$ for 340 seconds for the $512 \times 0.5 \mathrm{MHz}$ system, and a $50 \mu \mathrm{s}$ sample time was used for 450 seconds for the other system.

Data processing consisted of sub-band dedispersion, fine dedispersion and periodicity search. As shown in Figure 1, our search is very sensitive to submillisecond pulsars up to a dispersion measure of $\mathrm{DM}=80 \mathrm{pc} \mathrm{cm}{ }^{-3}$, much better than the Parkes $70 \mathrm{~cm}$ pulsar survey (Manchester et al. 1996) which had a sensitivity of $3 \mathrm{mJy}$ at $400 \mathrm{MHz}$ for long period pulsars, corresponding to about $0.5 \mathrm{mJy}$ at $1400 \mathrm{MHz}$ for a typical pulsar spectral index of -1.5 . That survey was insensitive to pulsars with period less than $2 \mathrm{~ms}$ for typical DMs.

No pulsars were discovered though observations of known pulsars gave results as expected. This result implies that either radio pulsars do not have pulse periods of less than $1 \mathrm{~ms}$, or submillisecond pulsars are not highly polarized.

\section{References}

Condon, J. J., Cotton, W. D., Greisen, E. W., Yin, Q. F., Perley, R. A., Taylor, G. B., \& Broderick, J. J. 1998, AJ, 115, 1693

Edwards, R. T., van Straten, W., \& Bailes, M. 2001, ApJ, 560, 365

Han, J. L., \& Tian, W. W. 1999, A\&AS 136, 571

Madsen, J. 1998, Phys. Rev. Lett., 81, 3311

Manchester, R. N., et al. 1996, MNRAS, 279, 1235 